\title{
Evaluation of knowledge of cancer pain management among medical practitioners in a low-resource setting
}

This article was published in the following Dove Press journal:

Journal of Pain Research

5 February 2013

Number of times this article has been viewed

\author{
EO Ogboli-Nwasor ${ }^{1}$ \\ JG Makama² \\ LMD Yusufu²
}

'Department of Anesthesia, Ahmadu Bello University Teaching Hospital, Shika, Zaria, Nigeria; ${ }^{2}$ Department of Surgery, Ahmadu Bello University Teaching Hospital, Shika, Zaria, Nigeria
Correspondence: Elizabeth OgboliNwasor

Department of Anesthesia, Ahmadu Bello University Teaching Hospital,

Shika, Zaria, Nigeria

Tel +23480370l 0020

Email drnwasor@yahoo.com
Background: Several factors considered to be barriers to cancer pain management have been reported in the past. The knowledge of cancer pain management may be a hindrance to the proper assessment and treatment of pain in cancer patients.

Objective: This report presents an evaluation of the knowledge and practice of cancer pain management among medical practitioners in Ahmadu Bello University Teaching Hospital, Shika, Zaria.

Methods: This report involves medical practitioners at the Ahmadu Bello University Teaching Hospital who are directly involved in the management of cancer patients. Information was obtained using a structured questionnaire, and the data were analyzed using SPSS (version 11.5).

Results: The response rate to the questionnaire was $82 \%$, with an age range of 23 to 50 years (mean age, 34.9), and the majority of actual respondents, 55 (67\%), were male. Thirty-six (44\%) strongly agreed that cancer patients require pain relief. Yet only $40 \%$ of the respondents routinely conducted pain assessments among cancer patients, while $51 \%$ only treated when patients complained of pain. Concerning the type of analgesic commonly used for cancer patients, $43 \%$ used weak opioids, $32 \%$ used NSAIDs, and only $20 \%$ used strong opioids. Seventy-five respondents $(91.5 \%)$ had no formal training on pain management.

Conclusion: The knowledge of pain management for cancer patients among medical personnel at the Ahmadu Bello University Teaching Hospital appears to be elementary. We recommend that formal training in the form of lectures, seminars, and workshops on cancer pain management should be part of continuing medical education in low-resource settings like the Ahmadu Bello University Teaching Hospital.

Keywords: cancer pain, management, evaluation, knowledge, medical practitioners

\section{Introduction}

The prevalence and severity of pain among cancer patients has notably increased in recent years. ${ }^{1-3}$ Experts in pain management have estimated that a significant number of all cancer patients die without adequate pain relief, despite the fact that the tools for adequate pain control are available. ${ }^{2}$ Several factors considered to be barriers to cancer pain management have already been reported, ${ }^{4}$ and the physician's knowledge of cancer pain management has been rated highly among those factors. ${ }^{1,4}$ With the appropriate anticancer therapies, opioids drugs, nerve blocks, and other techniques, such as psychosocial care, satisfactory pain relief could be achieved for most cancer patients. Although the World Health Organization (WHO) analgesic ladder is effective for more than $80 \%$ of cancer pain, the addition of appropriate adjuvant drugs, along with early intervention, is 
needed for an improved and prolonged quality of life (QoL). Effective cancer pain treatment requires a holistic approach, with timely assessments, measurements of pain, an understanding of the pathophysiology involved in causing particular types of pain, and an understanding of drugs to relieve particular types of pain. Sustaining the effort of intervention is also necessary. The aim of this report is to evaluate the knowledge of cancer pain management among medical practitioners in our center.

\section{Materials and methods}

Ahmadu Bello University Teaching Hospital is a tertiary teaching hospital located in the north-western part of Nigeria. This hospital is a referral center and plays a major role in health care delivery in this part of the country. We carried out a study that sought to evaluate the knowledge of cancer pain management among medical practitioners, using a structured questionnaire to obtain the necessary information (Figure S1). The questionnaire was drafted by one of the authors (JGM), critically reviewed by the co-authors (LMDY and EON), pre-tested, and validated using a population similar to the target population.

It was evident that a subset of the target population and a similar independent population both clearly understood the questionnaire's information. The questionnaire was then administered by one of the authors (JGM). The questionnaire was administered, face to face, to all the doctors directly involved in the management of cancer patients at the Ahmadu Bello University Teaching Hospital. The medical practitioners read the questionnaire and ticked the appropriate boxes (or wrote their responses where applicable). The population of medical practitioners was composed of house officers, medical officers, registrars, senior registrars, and consultants. Newly employed medical practitioners, those who had not spent a minimum of 3 months in the hospital, and/or those that were not directly involved in the management of cancer patients were excluded from the study.

The questionnaire utilized both open- and closed-ended questions to determine whether respondents considered pain to be a significant health problem among cancer patients. The questionnaire was also designed to determine how frequently the physician attended to cancer patients and whether he or she made a routine assessment of pain in these patients. The most appropriate modality of cancer pain control and the commonly used form of analgesic for cancer patients was also determined. Respondents were also asked whether they would consider changing the strength of the analgesic used for a patient or leave the patient alone if the first attempt at abating the pain failed. Limitations to cancer pain management were also determined and whether the respondents have had formal training on pain management. Statistical data analysis was carried out using SPSS (version 11.5, IBM Corporation, Armonk, NY, USA). Simple frequencies were determined for variables. Where multiple responses were required, each response was treated as a separate variable.

\section{Results}

Eighty-two (82) medical practitioners responded to the questionnaire, aged 23 to 50 years (mean, 34.9). There were $55(67.1 \%)$ males and $27(32.9 \%)$ females. The majority of the medical practitioners assessed were surgical residents (Figure 1). Thirty-six (43.9\%) strongly agreed that cancer patients require pain relief; while 27 (32.9\%) agreed, ten $(12.2 \%)$ were undecided, six (7.3\%) disagreed, and three (3.7\%) strongly disagreed (Figure 2). Thirty-six (43.9\%) attended to cancer patients every day, 21 (25.6\%) attended to cancer patients twice weekly, $18(22.0 \%)$ attended weekly, and seven (8.5\%) attended monthly (Figure 3). Forty-nine $(59.8 \%)$ did not routinely make pain assessments among cancer patients, while 33 (40.2\%) did (Figure 4).

Concerning the most commonly used treatment protocol for pain relief in cancer patients, the survey results revealed that $42(51.2 \%)$ of the respondents treated for pain only when patients complained (Table 1). The types of analgesics commonly used for cancer patients were weak opioids, 38 (43.3\%); non-steroidal anti-inflammatory drugs (NSAIDs), 26 (31.7\%); and strong opioids, 16 (19.5\%); while two $(2.4 \%)$ of the respondents used other modalities (Figure 5). Concerning the action to be taken if the first attempt at treating cancer pain failed, 36 (43.9\%) of the respondents would give stronger analgesics, $29(35.4 \%)$ would combine stronger and mild analgesic, 14 (17.1\%) would reassure the patient, and three (3.7\%) would use a non-drug modality (Figure 6).

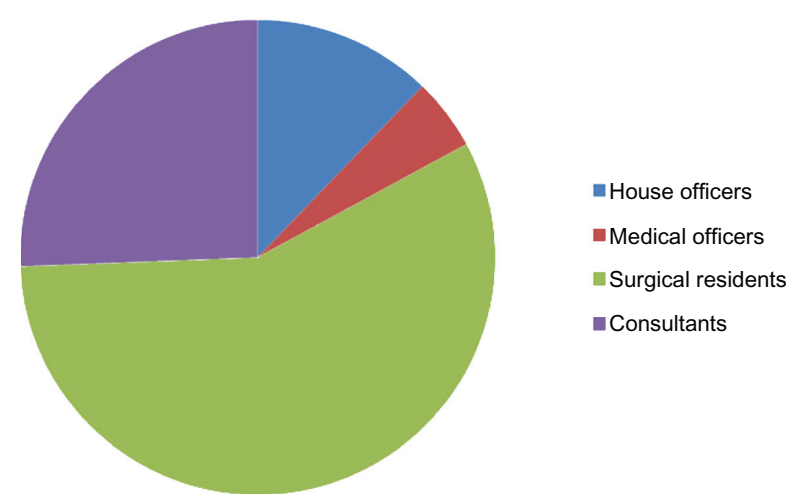

Figure I The categories of medical practitioners assessed. 


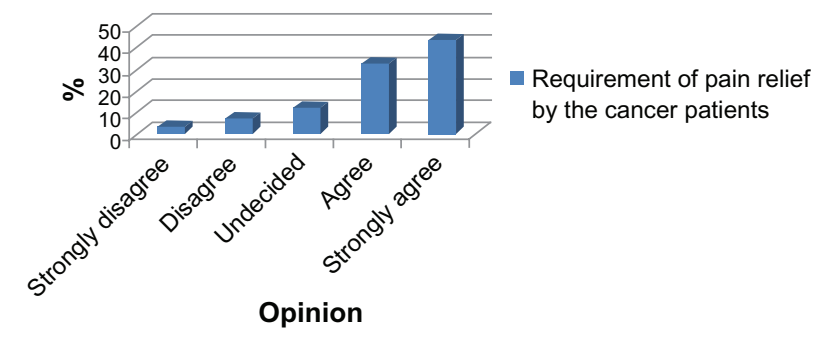

Figure 2 Whether cancer patients require pain relief medication.

In the case of perceived limitations to adequate cancer pain management, 44 (53.7\%) of the respondents felt that they had poor knowledge of cancer pain management, 18 (22.\%) were of the opinion that patients arrive at the hospital with already advanced stages of cancer, $13(15.9 \%)$ were afraid of serious drug side effects, while seven $(8.5 \%)$ reported the non-availability of stronger analgesic drugs (Figure 7). When respondents were asked whether they have had any formal training on pain management, $75(91.5 \%)$ had no formal training, while only seven ( $8.5 \%$ ) had received some formal training on pain management.

\section{Discussion}

In the past years, tremendous progress has been made in the understanding of the mechanisms of cancer pain. ${ }^{1-4}$ Cancer pain, affecting $50 \%$ of patients at any given cancer stage and $75 \%$ of patients with advanced cancers, ${ }^{5}$ has been studied and defined in relation to the following: causes neoplastic disease, $60 \%-80 \%$; therapies, $25 \%-20 \%$; not associated with either disease or therapy, $10 \%-5 \%$; pathophysiological characteristics (nociceptive pain, 50\%-70\%; neuropathic pain, $10 \%-30 \%$; mixed pain, $20 \%-40 \%$ ); intensity (severe pain in $30 \%$ of cases); and the number of pain sites (one pain site, 20\%; 2-4 pain sites, $60 \%$; and $>4$ pain sites, $20 \%) .{ }^{6-8}$

The pathogenesis of malignancy-associated pain is often heterogeneous, and a significant percentage of cancer patients have pain that is produced by multiple mechanisms. Thus,

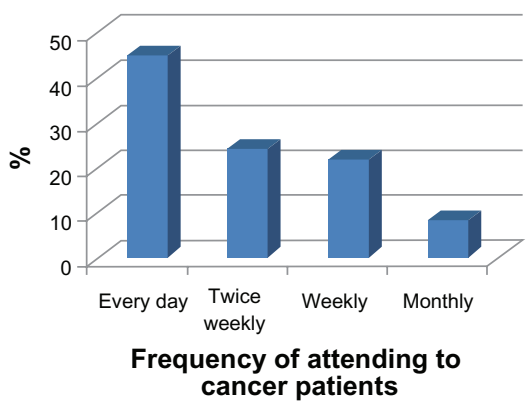

Figure 3 Attending to cancer patients.

Attending to cancer patients



Figure 4 Assessment of pain in cancer patients.

an understanding of the pathophysiology of cancer pain may have therapeutic implications and may influence the selection of pharmacological and non-pharmacological treatments. The WHO three-step analgesic ladder has been the gold standard for the therapy of cancer pain. ${ }^{2}$ However, a treatment approach that determines the sequence of analgesic therapies, based primarily on an individualized therapeutic course for cancer pain, will inevitably replace the epidemiologically based approach of the WHO three-step ladder's measure of pain intensity.

Replacing the WHO three-step ladder with a multifaceted approach is linked to the most progressive scientific thinking. As an understanding of the specific cellular mechanisms of cancer pain increases, more effective therapies can be developed, targeting the precise mediators of pain according to both the nature of a specific tumor and the individual suffering from pain. ${ }^{10}$ Several of the specific mediators of cancer pain have been identified. ${ }^{10,11}$ It has been discovered that certain tumors release cancer pain mediators, such as cyclo-oxygenase-2 (COX-2), peptide endothelin-1, and malignancy-associated acidosis, which sensitize or stimulate primary afferent neurons. ${ }^{10,11}$

It is essential to perform a comprehensive evaluation of pain in cancer patients. ${ }^{12,13}$ The severity of cancer pain is dynamic, often fluctuating as the disease progresses and as different therapies are administered. ${ }^{14}$ Therefore, it is necessary to evaluate and re-evaluate the patient's pain in order to determine the severity on a serial basis. ${ }^{15-17}$ This therefore becomes a necessity, that routine assessment of pain in cancer patients should be done. In our report, only $40 \%$ of the medical practitioners in Ahmadu Bello University Teaching Hospital

Table I Treatment protocols for cancer patients

\begin{tabular}{lll}
\hline S/no & Type of protocol & No of respondents (\%) \\
\hline I & Treat only when patient complains & $42(5 I .2)$ \\
2 & Give regular pain medications & $27(32.9)$ \\
3 & Use of other non-drug modalities & $9(11.0)$ \\
4 & Reassurance & $4(4.9)$ \\
\hline
\end{tabular}




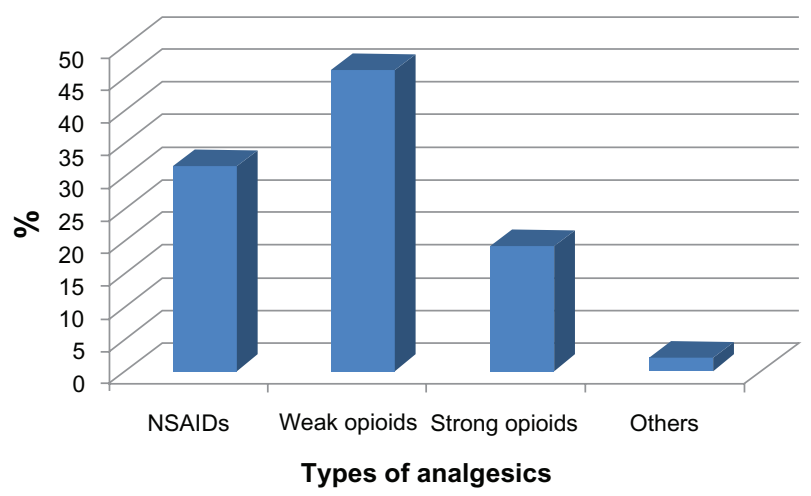

Figure $\mathbf{5}$ Types of analgesia used in cancer patients. Abbreviation: NSAIDs; non steroidal anti-inflammatory drugs.

admitted to performing routine assessments of pain among their cancer patients (Figure 4). A survey by Soyannwo et al among surgeons in West Africa reported that pain assessment was mostly by the verbal rating scale, and only $20 \%$ included psychological measurements in their schedules. ${ }^{18}$

Our survey results revealed that $51.2 \%$ of the respondents treated for pain only when patients complained (Table 1). This approach to pain management should be discouraged as much as possible, and routine pain assessments, using either the verbal rating scale or the visual analogue scale, should be encouraged. In our study, the types of analgesics commonly used for cancer patients were weak opioids, such as tramadol and DF118 (43\%), alone or in combination with NSAIDs $(32 \%)$. These are usually administered orally. Strong opioids, such as oral or parenteral morphine, pethidine, and pentazocine were used by only $19.5 \%$ of the respondents, while $2.4 \%$ of the respondents used other modalities (Figure 5).

While the correct use of the WHO analgesic ladder results in successful pain management in $90 \%$ of patients, ${ }^{19}$ a number of studies have reported inadequate pain control in $40 \%-70 \%$ of patients, ${ }^{15,20}$ resulting in the emergence of a new type of epidemiology, that of "failed pain control," caused by a series of obstacles preventing adequate cancer pain management. Thus, the current thinking is focused on the combination of therapeutic agents, particularly in situations of "failed pain

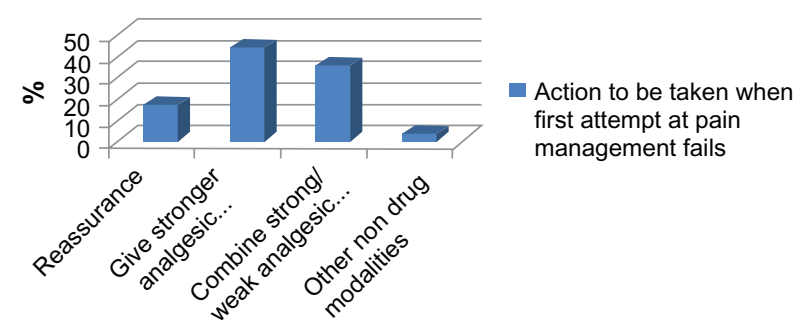

Action to be taken

Figure 6 Action to be taken when the first attempt at pain management fails.

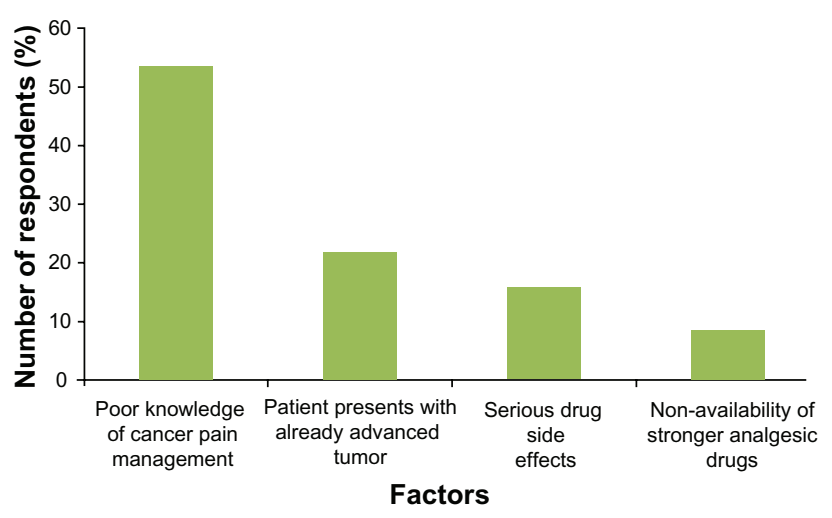

Figure 7 Perceived limitations to adequate cancer pain management.

control." An in-depth understanding of the pathophysiology and the various cellular mechanisms of pain in cancer patients has lent support to the use of combined therapeutic agents..$^{14,15}$ Some cancer patients have responded well to a combination of opioids and antidepressants. Other combinations have been tremendously successful, including opioids and corticosteroids, opioids and anticonvulsants, and opioids and NSAIDs. The medical practitioners in our study may not have been conversant with these combinations, which may be why they did not report the use of these combination therapies.

Concerning the action to be taken if the first attempt at treating cancer pain failed, $44 \%$ of the respondents would give stronger analgesics, $35 \%$ would combine stronger and mild analgesics, $17 \%$ would reassure the patient, and $4 \%$ would use a non-drug modality, such as physiotherapy or psychotherapy (Figure 6). Thus, our findings suggest that the majority of the medical practitioners in this survey $(79 \%)$ tend to follow the WHO analgesic ladder, with some practicing multimodal pain management. The practice of giving stronger analgesics may seem to be good, but this may also lead to increased side effects and may encourage opioid tolerance. Consequently, it may be preferable to combine a strong and mild analgesic in order to achieve better pain relief in cancer patients. The doses of the analgesic should be titrated against the patient's pain, gradually increasing until the patient is comfortable. ${ }^{21}$ Each dose should also be given before the effect of the previous dose has fully worn off. That way, it is possible to relieve pain continuously, though rescue doses for breakthrough pain may have to be given at intervals in addition to the regular pain treatment schedules. ${ }^{21}$

Although other sophisticated modes of delivery are available in developed countries, we found that most respondents in our study used opioids and NSAIDs via the oral and intramuscular routes only. This finding is similar to an earlier study by Amanor-Boadu et al among surgical residents at the University College Hospital, Ibadan, in south-western 
Nigeria. ${ }^{22}$ They concluded that the residents' knowledge of cancer pain management was deficient, and they recommended a workshop approach for training on cancer pain management. Consequently, concerning the perceived limitations to adequate cancer pain management, $54 \%$ of our respondents felt that there was poor knowledge of cancer pain management among medical practitioners. This is a surprising admission among medical practitioners who are expected to be very knowledgeable about all the modalities of patient management. Some of the respondents (22\%) were of the opinion that patients arrive at the hospital with already advanced stages of cancers and, as such, the respondents view this late presentation as a barrier to adequate cancer pain management because there is very little time to achieve optimal pain control. This, however, should not be the case. Even if a patient presents with an advanced tumor, potent analgesics should be offered to the patient to achieve optimal pain relief in the shortest possible time.

In our study, while $16 \%$ of the respondents were afraid of serious drug side effects, about $9 \%$ of the respondents reported the non-availability of stronger analgesic drugs (Figure 7). The fear of opioid side effects seems to be a common limitation to the use of opioids. The most commonly feared side effects are usually respiratory depression, as an immediate side effect of parenteral opioids, and opioid addiction with prolonged use on patients. This "opioid phobia" or "opiophobia" may be a major hindrance to adequate cancer pain management, as seen in $16 \%$ of the respondents in our survey.

Barriers to the correct treatment of cancer pain have been identified in recent years, and the Agency for Health Care Policy and Research (AHCPR) classified them into three categories: system, professional, and patient barriers. ${ }^{23}$ System barriers include the low priority given to cancer pain treatment and the legal and regulatory obstacles to the use of opioids for cancer pain. Cancer patients run the risk of becoming innocent victims in a war waged against opioid abuse and addiction if the norms regarding the two kinds of use (therapeutic or nontherapeutic) are not clearly distinguished. Furthermore, health professionals may be worried about regulatory scrutiny and may opt not to use opioid therapy for this reason. System barriers can also be internal (such as the inadequate or late start-up of palliative care programs) or external (such as regulatory excesses and complications). In developing countries, system barriers may also include "opiophobia," the high cost of opioids, and the problems of geographic dispersion, availability for treatment, and access to opioids. ${ }^{6,24,25}$
It has been reported that physician barriers to correct cancer pain management are numerous. ${ }^{6,24}$ These include the use of a "disease-based" rather than a "symptom-based" model of care, the lack of physician education and the failure to follow existing guidelines, and the lack of priority given to symptom management. Another professional barrier is the establishment of an analgesic level based on the prognosis rather than the severity of pain, and thus, the poor assessment of pain and the lack of proactive questioning about symptoms. Likewise, insufficient experience with pain management (poor knowledge of opioid pharmacology, conversion, equianalgesia, rotation, doses, and ratios for breakthrough pain drugs) is a professional barrier, as is the fear of patient addiction and analgesic tolerance, the failure to use adjuvants, the failure to consider or to treat opioid side effects, the failure to document information on drugs used, dosages given, timing, breakthrough pain, and laxatives administered, and the failure to follow-up.

Our findings suggest that the lack of physician education or inadequate knowledge about cancer pain management plays a major role as a hindrance to adequate cancer pain management. This is because $91.5 \%$ of the respondents reported that they had no formal training, while only $8.5 \%$ had received some formal training on pain management. The system barrier of opioid unavailability also acts as a hindrance to adequate cancer pain management, though to a lesser extent, as reported by $9 \%$ of the respondents in this survey.

\section{Conclusion}

There is inadequate knowledge about the current trends in cancer pain management among the medical practitioners surveyed. Although most of the respondents seemed to use the WHO analgesic ladder, the majority of the medical practitioners surveyed had no formal training on cancer pain management. We recommend that pain management should be part of the curriculum in all medical schools. This formal training should take the form of lectures, seminars, and workshops on cancer pain management.

\section{Disclosure}

The authors have no conflicts of interest to disclose.

\section{References}

1. Foley KM, Arbit E. Management of cancer pain. In: Devita VT, Hellman S, Rosenberg SA, editors. Cancer: Principles and Practice of Oncology, 3rd ed. Philadelphia: Lippincott; 1989:2064-2087.

2. World Health Organization. Cancer Pain Relief: With a Guide to Opioid Availability, 2nd ed. Geneva: World Health Organization; 1996. 
3. van den Beuken-van Everdingen MH, de Rijke JM, Kessels AG, Schouten HC, van Kleef M, Patijn J. High prevalence of pain in patients with cancer in large population-based study in The Netherlands. Pain. 2007; 132:312-320.

4. Cleeland CS, Cleeland LM, Reuven Dar MS, Rinehardt LC. Factors influencing physician management of cancer pain. Cancer. 1986;58:796-800

5. Hearn J, Higginson IJ. Cancer pain epidemiology: a systematic review. In: Bruera ED, Portenoy RK, editors. Cancer Pain: Assessment and Management. Cambridge (UK): Cambridge University Press; 2003:19-37.

6. Davis MP, Walsh D. Epidemiology of cancer pain and factors influencing poor pain control. Am J Hosp Palliat Med. 2004;21:137-142.

7. Foley K. Acute and chronic cancer pain syndromes. In: Doyle D, Hanks G, Cherny N, Calman K, editors. Oxford Textbook of Palliative Medicine 3rd Edition. Oxford (UK): Oxford University Press; 2004:298-316.

8. Caraceni A, Portenoy RK. An international survey of cancer pain characteristics and syndromes. IASP Task Force on Cancer Pain. International Association for the Study of Pain. Pain. 1999;82:263-274.

9. Esphani N, Bruera E. Current trends in cancer pain management. In: De Leon-Casasola OA, editor. Cancer Pain: Pharmacological, Interventional, and Palliative Approaches. Philadelphia: Saunders Elsevier; 2006:17.

10. Lee BN, Dantzer R, Langley KE, et al. A cytokine-based neuroimmunologic mechanism of cancer-related symptoms. Neuroimmunomodulation. 2004;11:279-292.

11. Sabino MA, Ghilardi JR, Jongen JL, et al. Simultaneous reduction in cancer pain, bone destruction, and growth by selective inhibition of cyclooxygenase-2. Cancer Res. 2002;62:7342-7349.

12. Gonzales GR, Elliot KJ, Portenoy RK, Foley KM. The impact of a comprehensive evaluation in the management of cancer pain. Pain. 1991;47:141-144.

13. Ventafridda V. Continuing care: a major issue in cancer pain management. Pain. 1989;36:137-143.
14. Cherny NI, Portenoy RK. The management of cancer pain. CA cancer J Clin. 1994;44:262-303.

15. Von Roenn JH, Cleeland CS, Gonin R, Hatfield AK, Pandya KJ. Physician attitudes and practice in cancer pain management. A survey from the Eastern Cooperative Oncology Group. Ann Intern Med. 1993;119:121-126.

16. Baillie L. A review of pain assessment tools. Nurs Stand. 1993;7:25-29.

17. Russel PB, Aveyard SC, Oxenham DR. An assessment of methods used to evaluate the adequacy of cancer pain management. J Pain Symptom Manage. 2006;32:581-588.

18. Soyannwo A, Amanor-Boadu SD. Management of cancer pain-a survey of current practice in West Africa. Niger Postgrad Med J. 2001;8: $175-178$.

19. Meuser T, Pietruck C, Radbruch L, Stute P, Lehmann KA, Grond S. Symptoms during cancer pain treatment following WHO-guidelines: a longitudinal follow-up study of symptom prevalence, severity and etiology. Pain. 2001;93:247-257.

20. Zenz M, Zenz T, Tryba M, Strumpf M. Severe undertreatment of cancer pain: a 3-year survey of the German situation. J Pain Symptom Manage. 1995;10:187-191.

21. Kanner RM, Foley KM. Patterns of Narcotic drug use in a cancer pain clinic. Ann NY Acad Sci. 1981;362:161-172.

22. Amanor-Boadu SD, Adebamowo CA, Olubowale A, Soyanwo OA. Evaluation of surgical resident staff knowledge of cancer pain: assessment and treatment. Niger J Clin Pract. 2002;5:10-13.

23. Jacox A, Carr DB, Payne R. Management of Cancer Pain: AHCPR Clinical Practice Guidelines, No. 9. Rockville (MD): Agency for Health Care Policy and Research; 1994.

24. Sun VC, Borneman T, Ferrell B, Piper B, Koczywas M, Choi K. Overcoming barriers to cancer pain management: an institutional change model. J Pain Symptom Manage. 2007;34:359-369.

25. Dahl JL. How to reduce fears of legal/regulatory scrutiny in managing pain in cancer patients. J Support Oncol. 2005;3:384-388. 


\section{Supplementary figure}

\section{Introduction}

Our group is studying the experience of doctors in offering pain relief for cancer patients. Based on the findings, this research has the aim of making suggestions that will improve the practice of analgesia in cancer patients in our environment. Your response will be treated with utmost confidentiality and will be highly appreciated.

1. Age -----

2. Sex ( ) Male ( ) Female

3. Department ( ) Anaesthesia ( ) Laboratory medicine ( ) Medicine ( ) O and G ( ) Paediatric ( ) Surgery ( ) Others (specify)

4. Highest qualification ( ) MB;BS ( ) Primary ( ) Part I ( ) Part II ( ) Msc ( ) PhD ( ) FWACS ( ) FNMC ( ) ( ) Others (Specify)

5. Status ( ) House officer ( ) Medical officer ( ) Resident Doctor ( ) Consultant ( ) Others (Specify) -------

6. Post-graduation experience (in years) ( ) <1 ( ) 1-5 ( ) 6-10 ( ) 11-15 ( ) >15

7. How often do you attend to cancer patients? ( ) Everyday ( ) Twice a week ( ) Weekly ( ) Monthly

8. Do cancer patients require pain relief ( ) Strongly disagree ( ) Disagree ( ) Undecided ( ) Agree ( ) strongly agree

9. Do you routinely make pain assessment of cancer patients ( ) Yes ( ) No

10. Method of pain assessment ( ) None ( ) Visual analogue ( ) Linear analogue ( ) Verbal ( ) Others (Specify)

11. In a patient with cancer, the most appropriate pain control protocol is ( ) reassurance ( ) treat only when patient complains ( ) regular medications ( ) others (specify)

12. Which type of analgesic agents do you use for cancer patients commonly ( ) None ( ) NSAIDs ( ) Weak opioids ( ) Strong opioids ( ) Others (Specify)

13. What will be your action following failure of the first attempt at treating cancer pain ( ) Leave patient alone ( ) Reassure him/her ( ) Give a stronger analgesic ( ) Combine stronger and milder analgesic ( ) Use other non drug modalities.

14. Inadequate pain control in cancer patients is due to ( ) cancer pain is usually unresponsive to conventional analgesics ( ) Terminal illness ( ) Poor knowledge of cancer pain management ( ) Fear of side effects of analgesics particularly addiction.

15. Limitations to cancer pain management in our environment ( ) Poor knowledge of cancer pain management ( ) Side effects of analgesic drugs ( ) Non-availability of analgesic drugs ( ) Patients present with advanced tumours.

16. Any formal training/workshop/update course on pain management ( ) Yes ( ) No

\section{THANK YOU FOR YOUR PATIENCE AND TIME}

Figure SI The evaluation of knowledge of cancer pain management among medical practitioners.

Abbreviations: NSAID, nonsteroidal anti-in flammatory drug; MB, Bachelor of Medicine; BS, Bachelor of Surgery; FWACS, Fellow West African College of Surgeons; FNMC, Fellow National Postgraduate College (Nigeria).

\section{Publish your work in this journal}

The Journal of Pain Research is an international, peer-reviewed, open access, online journal that welcomes laboratory and clinical findings in the fields of pain research and the prevention and management of pain. Original research, reviews, symposium reports, hypothesis formation and commentaries are all considered for publication.
The manuscript management system is completely online and includes a very quick and fair peer-review system, which is all easy to use. Visit http://www.dovepress.com/testimonials.php to read real quotes from published authors. 